\title{
Age-Related Alterations of Nonalcoholic Steatohepatitis in Sprague-Dawley Rats Fed a High-Fat and High-Cholesterol Diet
}

\author{
Katsuhisa OMAGARI ${ }^{1,2}$, Eri ASAKAWA ${ }^{2}$, Marin SASAO ${ }^{2}$, Sumire NARITA ${ }^{2}$, \\ Mei HISANO ${ }^{2}$, Ayumi FuKUdA ${ }^{1}$, Kazuhito SuRugA ${ }^{1,2}$, \\ Mayuko ICHIMURA ${ }^{3}$ and Koichi TSUNEYAMA ${ }^{3}$ \\ ${ }^{1}$ Division of Nutrition Science, Graduate School of Human Health Science, \\ University of Nagasaki, Siebold, Nagasaki 851-2195, Japan \\ ${ }^{2}$ Department of Nutrition, Faculty of Nursing and Nutrition, University of Nagasaki, \\ Siebold, Nagasaki 851-2195, Japan \\ ${ }^{3}$ Department of Pathology and Laboratory Medicine, Tokushima University Graduate School, \\ Tokushima 770-8503, Japan \\ (Received January 25, 2019)
}

\begin{abstract}
Summary Nonalcoholic steatohepatitis (NASH), a subtype of nonalcoholic fatty liver disease (NAFLD), has a potentially progressive course that can lead to liver cirrhosis. Age is strongly associated with the development and progression of NAFLD/NASH, but the natural history of pediatric NAFLD is still not fully understood. Here, we evaluated the age-related alterations of NASH in 5-, 9- and 13-wk-old male Sprague-Dawley rats that were fed a highfat and high-cholesterol diet (30\% fat, $1.25 \%$ cholesterol and $0.5 \%$ sodium cholate, $\mathrm{w} / \mathrm{w}$ ) for $9 \mathrm{wk}$ ( 6 rats/group). Our results showed that the cumulative energy intake, body weight gain and food efficacy during the 9-wk rearing period were highest in the youngest group and lowest in the oldest group. Serologically, almost all parameters including the serum triglyceride and total cholesterol were similar regardless of age. Histopathological findings, such as hepatic steatosis, lobular inflammation and hepatocyte ballooning, were also similar regardless of age, but hepatic fibrosis was more evident in the oldest group. Also, the mRNA expression levels of some fibrogenic, inflammatory, oxidative stress and cholesterol or lipid metabolism-related genes in the liver were highest in the oldest group and lowest in the youngest group, although the difference was not statistically significant. These results indicated that aging is likely associated with the development of NASH. Because the cumulative energy intake and daily food intake/body weight were not similar among groups in the present study, further studies designed with an equivalent daily food intake/body weight among groups are needed in order to interpret the exact nutritional effect.
\end{abstract}

Key Words nonalcoholic steatohepatitis, high-fat high-cholesterol diet, age, hepatic fibrosis, rat

Due to the increasing prevalence of obesity and type 2 diabetes mellitus in children and adults, the prevalence of nonalcoholic fatty liver disease (NAFLD) has also been increasing worldwide. NAFLD is not always benign, and nonalcoholic steatohepatitis (NASH), a subtype of NAFLD, has a potentially progressive course that can lead to liver cirrhosis and/or hepatocellular carcinoma $(1,2)$.

Aging is associated with a physiological increase in lipid accumulation in non-adipose tissues, including the liver (3), age is therefore strongly associated with the development and progression of NAFLD/NASH (4). On the other hand, pediatric NAFLD has been reported to be becoming more prevalent. It is the most common cause of liver disease in children, and is more prevalent in those with metabolic syndrome (5). The prevalence of NAFLD is estimated to be $3 \%$ to $12 \%$ in the general pediatric population $(1,5)$, and $34.2 \%$ in children attend-

E-mail: omagari@sun.ac.jp ing child obesity clinics (6). The major risk factors for NAFLD in children include obesity, unhealthy eating habits and a sedentary lifestyle (7). Therefore, lifestyle intervention counseling, including assessments of the quality of diets and caloric intake by a registered dietitian, can be useful (5). Diets and regular physical activity play a key role in preventing and treating NAFLD (7).

Results of animal studies analyzing the role of diet in the development of NASH suggest that a high-fat and high-cholesterol (HFC) diet is critical for the onset of NASH (8). In this context, we recently reported that the administration of a HFC diet to 9-wk-old male SpragueDawley (SD) rats induced NASH with advanced fibrosis within a relatively short period of 9 or $18 \mathrm{wk}(9,10)$. In the present study, we evaluated the age-related alterations of NASH in 5-, 9- and 13-wk-old male SD rats that were fed a HFC diet for $9 \mathrm{wk}$ by examining the serology, histopathology and the expression levels of fibrogenic, inflammatory, oxidative stress and cholesterol or lipid metabolism-related genes in the liver. 
Table 1. Proximate dietary composition.

Constituents

\begin{tabular}{lr}
\hline Water $(\mathrm{g})$ & 5.49 \\
Crude protein $(\mathrm{g})$ & 16.05 \\
Crude lipid $(\mathrm{g})$ & 3.54 \\
Crude ash $(\mathrm{g})$ & 4.03 \\
Crude fiber $(\mathrm{g})$ & 1.95 \\
Nitrogen free extract $(\mathrm{g})$ & 38.43 \\
Palm oil (g) & 28.75 \\
Cholesterol (g) & 1.25 \\
Sodium cholate (g) & 0.50 \\
\hline Total (g) & 100.00 \\
\hline Protein energy ratio (\%) & 12.63 \\
Lipid energy ratio (\%) & 57.15 \\
Carbohydrate energy ratio (\%) & 30.23 \\
\hline Energy (kcal/100 g) & 508.60 \\
\hline
\end{tabular}

\section{MATERIALS AND METHODS}

Animals and experimental design. Four-week-old (S group, $n=6$ ), 8-wk-old (M group, $n=6$ ), and 12-wk-old (L group, $n=6$ ) male SD rats were purchased from Japan SLC, Inc. (Hamamatsu, Japan) and housed individually in a temperature- and humidity-controlled room $\left(22-24^{\circ} \mathrm{C}\right.$ and $50-60 \%$ relative humidity) with a $12-\mathrm{h}$ light/dark cycle. After $1 \mathrm{wk}$ of acclimation with standard rodent chow (MF; Oriental Yeast Co., Ltd., Tokyo, Japan) and water ad libitum, the rats were fed a HFC diet for $9 \mathrm{wk}$. The proximate dietary composition of the HFC diet is shown in Table 1. The daily energy intake and body weight were monitored throughout the study. After the 9-wk rearing period, the rats at 14,18 , or $22 \mathrm{wk}$ of age were fasted for $8 \mathrm{~h}$ and sacrificed under anesthesia with pentobarbital sodium. Blood was collected from the inferior vena cava. The epididymal fat pad and liver were removed, washed in cold saline, and weighed. Liver tissues were either placed in $10 \%$ neutral buffered formalin or snap frozen in liquid nitrogen and stored at $-80^{\circ} \mathrm{C}$. All procedures performed on the animals were approved by the Animal Use Committee of University of Nagasaki (Approval No. 29-14), and the animals were maintained in accordance with the University of Nagasaki guidelines for the care and use of laboratory animals.

Serum biochemical analysis. Serum triglyceride (TG), free fatty acid (FFA), total cholesterol (TC) and glucose levels were determined using Triglyceride E test Wako, NEFA C test Wako, Cholesterol E test Wako, Free cholesterol E test Wako and Glucose C II test Wako (Wako Pure Chemical Industries, Ltd., Osaka, Japan), respectively. Serum aspartate aminotransferase (AST) and alanine aminotransferase (ALT) activities were determined using Transaminase C II test Wako (Wako Pure Chemical Industries, Ltd.). Serum insulin, leptin and adiponectin levels were measured using a rat insulin enzyme-
Table 2. Primer sequences for real-time reverse transcription polymerase chain reaction (Real-time RT-PCR).

\begin{tabular}{|c|c|}
\hline Primer & Sequence ( $5^{\prime}$ to $\left.3^{\prime}\right)$ \\
\hline \multirow[t]{2}{*}{ Col1a1 } & Forward: GCGTAGCCTACATGGACCAA \\
\hline & Reverse: AAGTTCCGGTGTGACTCGTG \\
\hline \multirow[t]{2}{*}{$\operatorname{Tgfb1}$} & Forward: CTTTGTACAACAGCACCCGC \\
\hline & Reverse: TAGATTGCGTTGTTGCGGTC \\
\hline \multirow[t]{2}{*}{ Acta2 } & Forward: GCCAAGAAGACATCCCTGAAGT \\
\hline & Reverse: TGTGGCAGATACAGATCAAGCAT \\
\hline \multirow[t]{2}{*}{$\operatorname{Tnf}$} & Forward: TGATCGGTCCCAACAAGGA \\
\hline & Reverse: TGGGCTACGGGCTTGTCA \\
\hline \multirow[t]{2}{*}{$\mathrm{Ccl} 2$} & Forward: TCTGTCACGCTTCTGGGCCTGT \\
\hline & Reverse: GGGGCATTAACTGCATCTGGCTGAG \\
\hline \multirow[t]{2}{*}{$I l 1 b$} & Forward: CTCCAGTCAGGCTTCCTTGTG \\
\hline & Reverse: GGTCATTCTCCTCACTGTCGAAA \\
\hline \multirow[t]{2}{*}{ Il6 } & Forward: GATACCACCCACAACAGACCAGTA \\
\hline & Reverse: TGCACAACTCTTTTCTCATTTCCA \\
\hline \multirow[t]{2}{*}{$N f k b$} & Forward: TGACATCATCAACATGAGAAACGA \\
\hline & Reverse: CCCCAACCCTCAGCAAGTC \\
\hline \multirow[t]{2}{*}{ Cyp2e1 } & Forward: CCCATCCTTGGGAACATTTTT \\
\hline & Reverse: GCCAAGGTGCAGTGTGAACA \\
\hline \multirow[t]{2}{*}{ Hmox1 } & Forward: CACAGGGTGACAGAAGAGGCTAA \\
\hline & Reverse: GGGACTCTGGTCTTTGTGTTCCT \\
\hline \multirow[t]{2}{*}{ Gpx1 } & Forward: GCTGCTCATTGAGAATGTCG \\
\hline & Reverse: GAATCTCTTCATTCTTGCCATT \\
\hline \multirow[t]{2}{*}{ Sod 2} & Forward: GACCTGCCTTACGACTATG \\
\hline & Reverse: TACTTCTCCTCGGTGACG \\
\hline \multirow[t]{2}{*}{ Nr1h3 } & Forward: CAGGACCAGCTCCAAGTAGA \\
\hline & Reverse: GAACATCAGTCGGTCGTGG \\
\hline \multirow[t]{2}{*}{ Mttp } & Forward: CAAGCTCAAGGCAGTGGTTG \\
\hline & Reverse: AGCAGGTACATCGTGGTGTC \\
\hline \multirow[t]{2}{*}{ Fasn } & Forward: CAACATTGACGCCAGTTCCG \\
\hline & Reverse: TTCGAGCCAGTGTCTTCCAC \\
\hline \multirow[t]{2}{*}{ Gpam } & Forward: GCTACCTGAAGGTGAGCCAG \\
\hline & Reverse: AGGTACTCAGACTCCGGGAC \\
\hline \multirow[t]{2}{*}{ Cpt1a } & Forward: AACCTCGGACCCAAATTGC \\
\hline & Reverse: GGCCCCGCAGGTAGATATATT \\
\hline \multirow[t]{2}{*}{$A \cos 1$} & Forward: CCACTGAACAAAACAGAGGTCC \\
\hline & Reverse: GTCCCAGGGAAACTTCAAAGC \\
\hline \multirow[t]{2}{*}{ Srebf1 } & Forward: CATGGACGAGCTACCCTTCG \\
\hline & Reverse: GAAGCATGTCTTCGATGTCGG \\
\hline \multirow{2}{*}{ Nr1h4 } & Forward: TGGGAATGTTGGCTGAATGTTTG \\
\hline & Reverse: TGCTAGCTTGGTCGTGGAG \\
\hline \multirow[t]{2}{*}{ RplpO } & Forward: GGTGTTTGACAATGGCAGCAT \\
\hline & Reverse: ATTGCGGACACCCTCTAGGA \\
\hline
\end{tabular}

linked immunosorbent assay (ELISA) kit (Morinaga Institute of Biological Science Inc., Yokohama, Japan), a mouse/rat leptin ELISA kit (Morinaga Institute of Biological Science Inc.) and a mouse/rat adiponectin ELISA kit (Otsuka Pharmaceuticals Co., Ltd., Tokyo, Japan), respectively.

Hepatic lipid analysis. Hepatic lipids were extracted from the frozen liver using the method of Folch et al. (11). The extract was dissolved in isopropanol and analyzed for TG and TC with a kit, as described above.

Histopathological assessment of the liver. After fixation in neutral buffered formalin, the liver tissues were embedded in paraffin, sectioned and stained with Azan 
Table 3. Cumulative energy intake, body weight, relative organ weights, serum parameters and hepatic lipid concentrations after the 9-wk rearing period.

\begin{tabular}{|c|c|c|c|}
\hline \multirow{2}{*}{ Item } & \multicolumn{3}{|c|}{ Group } \\
\hline & $S$ & M & $\mathrm{L}$ \\
\hline Cumulative energy intake (kcal) & $6,275 \pm 53^{\mathrm{a}}$ & $6,091 \pm 230^{\mathrm{a}}$ & $5,455 \pm 120^{\mathrm{b}}$ \\
\hline Final body weight (g) & $481 \pm 6$ & $504 \pm 22$ & $503 \pm 9$ \\
\hline Body weight gain $(\mathrm{g})$ & $319 \pm 6^{\mathrm{a}}$ & $206 \pm 19^{b}$ & $103 \pm 7^{\mathrm{c}}$ \\
\hline Food efficacy (g/kcal) & $0.0470 \pm 0.0044^{\mathrm{a}}$ & $0.0311 \pm 0.0035^{\mathrm{b}}$ & $0.0189 \pm 0.0008^{c}$ \\
\hline Liver weight/body weight (\%) & $6.00 \pm 0.23^{\mathrm{a}}$ & $5.43 \pm 0.10^{\mathrm{ab}}$ & $4.90 \pm 0.19^{\mathrm{b}}$ \\
\hline Epididymal fat pad weight/body weight (\%) & $2.09 \pm 0.30$ & $2.29 \pm 0.23$ & $1.99 \pm 0.18$ \\
\hline Serum triglyceride $(\mathrm{mg} / \mathrm{dL})$ & $74.0 \pm 11.8$ & $94.0 \pm 17.2$ & $88.0 \pm 8.7$ \\
\hline Serum free fatty acid $(\mathrm{mEq} / \mathrm{L})$ & $0.272 \pm 0.066$ & $0.275 \pm 0.044$ & $0.310 \pm 0.045$ \\
\hline Serum total cholesterol (mg/dL) & $76.3 \pm 9.7$ & $82.1 \pm 5.7$ & $77.8 \pm 9.2$ \\
\hline Serum glucose $(\mathrm{mg} / \mathrm{dL})$ & $164 \pm 10$ & $204 \pm 35$ & $167 \pm 8$ \\
\hline Serum insulin (ng/mL) & $2.07 \pm 0.47$ & $4.26 \pm 1.12$ & $3.54 \pm 0.67$ \\
\hline Serum leptin (ng/mL) & $9.40 \pm 1.88$ & $8.63 \pm 1.79$ & $6.88 \pm 1.18$ \\
\hline Serum adiponectin $(\mu \mathrm{g} / \mathrm{mL})$ & $4.22 \pm 0.30^{\mathrm{a}}$ & $3.10 \pm 0.12^{\mathrm{b}}$ & $2.93 \pm 0.17^{\mathrm{b}}$ \\
\hline Serum aspartate aminotransferase (IU/L) & $104.6 \pm 11.5$ & $77.9 \pm 9.0$ & $70.3 \pm 9.9$ \\
\hline Serum alanine aminotransferase (IU/L) & $20.0 \pm 2.4$ & $18.5 \pm 1.9$ & $19.5 \pm 1.2$ \\
\hline Hepatic triglyceride (mg/g tissue) & $86.9 \pm 8.1$ & $89.9 \pm 11.7$ & $92.7 \pm 18.1$ \\
\hline Hepatic total cholesterol (mg/g tissue) & $69.3 \pm 5.5$ & $52.1 \pm 3.3$ & $54.2 \pm 6.9$ \\
\hline
\end{tabular}

Values are expressed as means \pm SE. $n=6 /$ group.

${ }^{a b c}$ Values with different lowercase letters within a row are significantly different at $p<0.05$, as determined by one-way analysis of variance (ANOVA) and Tukey-Kramer's post hoc test.

Food efficacy was calculated as the body weight gain $(\mathrm{g}) /$ cumulative energy intake (kcal).

S, rats fed a high-fat and high-cholesterol (HFC) diet from 5 to 14 wk of age $(n=6)$; M, rats fed a HFC diet from 9 to 18 wk of age $(n=6)$; L, rats fed a HFC diet from 13 to 22 wk of age $(n=6)$.

as well as hematoxylin and eosin. All histopathological examinations were performed by a pathologist (K.T.) who was blinded to the experimental data. Histological findings from the liver were scored using the NASH Clinical Research Network Scoring System based on the following four semi-quantitative factors: steatosis (0-3), lobular inflammation (0-3), hepatocyte ballooning (0-2), and fibrosis (0-4). The NAFLD activity score (NAS) was defined as the unweighted sum of the scores for steatosis, lobular inflammation and hepatocyte ballooning. NAS scores $\geq 5$ and $\leq 2$ were considered to be diagnostic and not diagnostic, respectively, for steatohepatitis. Liver fibrosis (0-4) was also assessed according to this system (12). The scores for fibrosis was further classified as follows: a score of 0.5 represented scores between 0 and 1 ; and a score of 1.5 represented scores between 1 and 2 .

Quantification of mRNA using real-time reverse transcription polymerase chain reaction. Total RNA from the liver was extracted using RNAiso Plus (Takara Bio, Inc., Otsu, Japan) according to the manufacturer's instructions. RNA was reverse-transcribed to cDNA templates using a commercial kit (PrimeScript RT Master Mix, Takara Bio). Real-time reverse transcription polymerase chain reaction (Real-time RT-PCR) analysis was performed as described previously (9). Specific primers were designed using the primer-designing tool Primer-BLAST (National Center for Biotechnology Information [NCBI], Bethesda, MD, USA) and were synthesized by Greiner Bio-One Japan (Tokyo, Japan) (Table

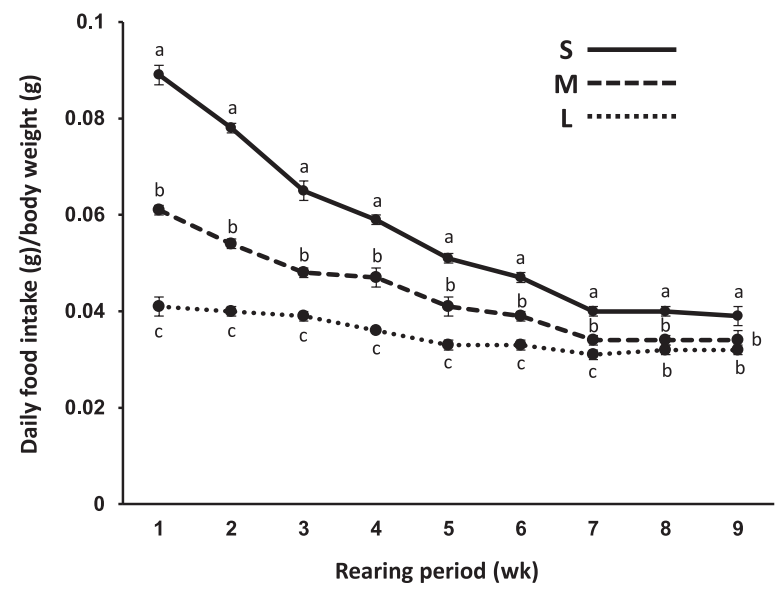

Fig. 1. Daily food intake (g)/body weight (g) changes during rearing period. ${ }^{\text {abc }}$ Values with different lowercase letters within a same rearing week are significantly different at $p<0.05$, as determined by one-way analysis of variance (ANOVA) and Tukey-Kramer's post hoc test. S, rats fed a high-fat and high-cholesterol (HFC) diet from 5 to $14 \mathrm{wk}$ of age $(n=6)$; M, rats fed a HFC diet from 9 to 18 wk of age $(n=6)$; L, rats fed a HFC diet from 13 to $22 \mathrm{wk}$ of age $(n=6)$.

2). The levels of mRNA relative to those of the internal control acidic ribosomal phosphoprotein (36B4) mRNA (RplpO) were determined using the $2-\Delta \Delta \mathrm{Ct}$ method. For studies in rats, the hepatic expression of genes involved in fibrosis [Col1a1 encoding collagen type I alpha 1 


\section{S}
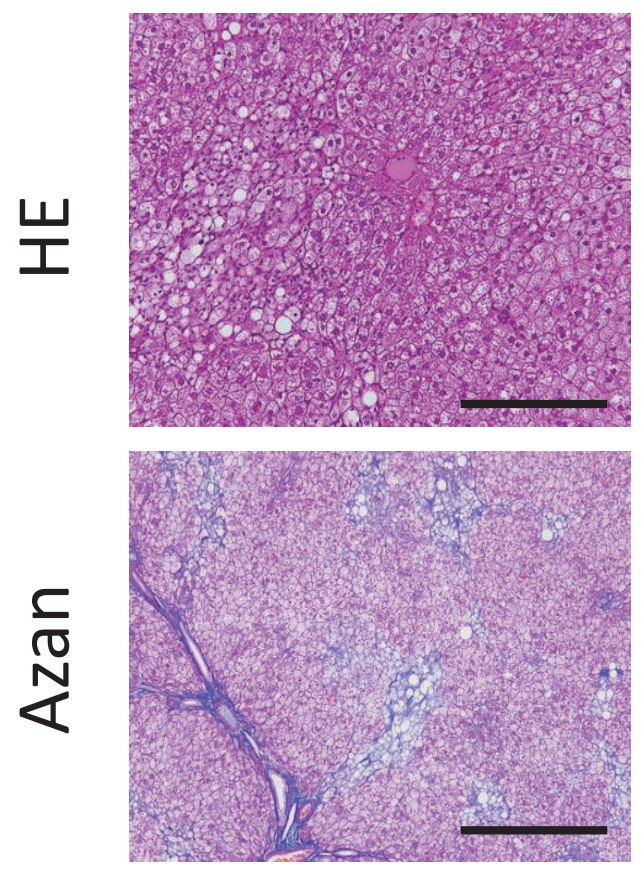

M
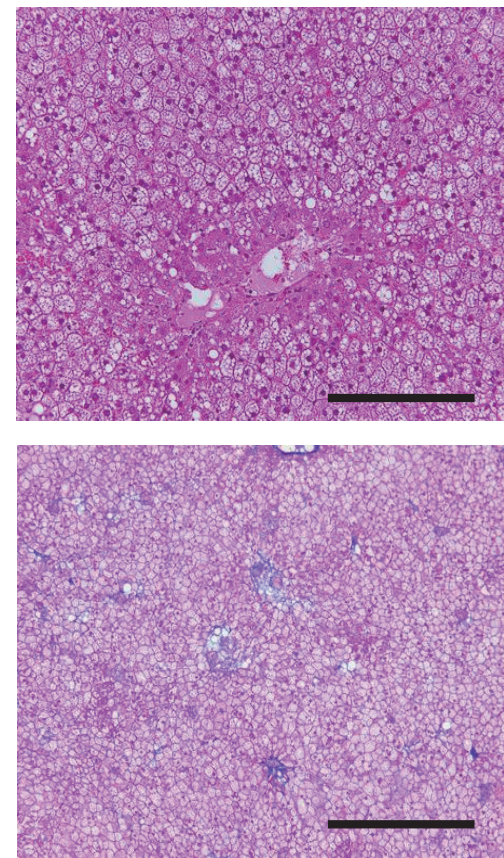

$\mathrm{L}$
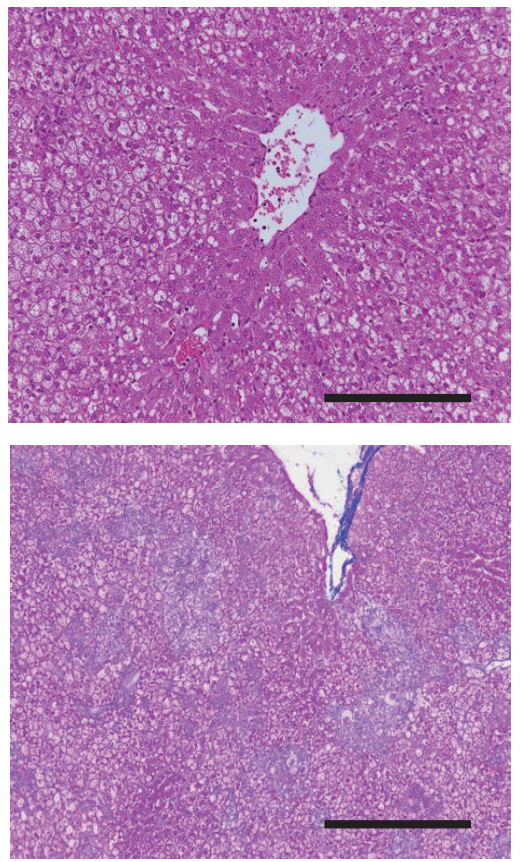

Fig. 2. Representative histopathological findings in the liver of the $S, M$ and L groups. In a rat of the $S$ group, severe steatosis (score 3), severe lobular inflammation (score 3) and no hepatocyte ballooning were observed. NAS score was 6 and perisinusoidal and portal/periportal fibrosis (score 2) was also observed. In a rat of the M group, severe steatosis (score 3), severe lobular inflammation (score 3) and no hepatocyte ballooning were observed. NAS score was 6 and mild perisinusoidal fibrosis (score 1) was also observed. In a rat of the L group, severe steatosis (score 3), severe lobular inflammation (score 3) and a few hepatocyte ballooning (score 1) were observed. NAS score was 7 and perisinusoidal and portal/ periportal fibrosis (score 2) was also observed. Hematoxylin and eosin-stained section: original maginification, 200×; scale bars $=200 \mu \mathrm{m}$. Azan-stained section: original maginification, $100 \times$; scale bars $=400 \mu \mathrm{m}$. S, rats fed a high-fat and high-cholesterol (HFC) diet from 5 to 14 wk of age $(n=6)$; M, rats fed a HFC diet from 9 to 18 wk of age $(n=6)$; L, rats fed a HFC diet from 13 to $22 \mathrm{wk}$ of age $(n=6)$.

(COL1A1), Tgfb1 encoding transforming growth factor$\beta$ (TGF- $\beta$ ) and Acta 2 encoding $\alpha$-smooth muscle actin $(\alpha$-SMA)], inflammation [Thf encoding tumor necrosis factor- $\alpha$ (TNF- $\alpha$ ), Ccl2 encoding monocyte chemoattractant protein-1 (MCP-1), Il1b encoding interleukin$1 \beta$ (IL-1 $\beta$ ), Il6 encoding interleukin-6 (IL-6) and Nfkb encoding nuclear factor $-\kappa \mathrm{B}(\mathrm{NF}-\kappa \mathrm{B})]$, oxidative stress [Cyp2e1 encoding cytochrome P450 family 2 subfamily E polypeptide 1 (CYP2E1), Hmox 1 encoding heme oxygenase-1 (HO-1), Gpx 1 encoding glutathione peroxidase-1 (GPX-1) and Sod 2 encoding manganese superoxide dismutase (MnSOD)], cholesterol metabolism [Nr1h3 encoding liver X receptor- $\alpha(\mathrm{LXR}-\alpha)]$ and lipid metabolism [Mttp encoding microsomal triglyceride transfer protein (MTP), Fasn encoding fatty acid synthase (FAS), Gpam encoding glycerol-3-phosphate acyltransferase 1 (GPAT1), Cpt1a encoding carnitine palmitoyltransferase-1 (CPT-1), Acox 1 encoding acyl-CoA oxidase (AOX), Srebf1 encoding sterol regulatory element-binding protein-1c (SREBP1c) and Nr1h4 encoding farnesoid X receptor $(\mathrm{FXR})]$ were quantified. All data were expressed as the fold-change relative to the S group.

Statistical analysis. All values were expressed as mean \pm standard error (SE). Differences between groups were tested for statistical significance using one-way analysis of variance (ANOVA), followed by TukeyKramer's post hoc test, or Fisher's exact probability test. Correlations between histopathological assessments of the liver (score) according to the NASH Clinical Research Network Scoring System (12) and the rat groups were determined by Spearman's rank correlation coefficient. All analyses were performed using IBM SPSS statistics software, version 24 (IBM, Chicago, IL, USA) on a computer with a Windows operating system. A $p$-value of less than 0.05 was considered to be statistically significant.

\section{RESULTS}

Cumulative energy intake, body weight and relative organ weights

As determined by ANOVA and Tukey-Kramer's post hoc test, the cumulative energy intake during the 9-wk rearing period was significantly lower in the L group than in the $\mathrm{S}$ and $\mathrm{M}$ groups $(p=0.005$ and 0.025 , respectively). Body weight gain and food efficacy, calculated as the body weight gain (g)/cumulative energy intake (kcal), were highest in the $\mathrm{S}$ group and lowest in the L group (Table 3). Daily food intake (g)/body weight (g) during the 9-wk rearing period gradually decreased in all three groups. This ratio was highest in the $S$ group 
Table 4. Histopathological assessment of the liver after the 9-wk rearing period according to the NASH Clinical Research Network Scoring System (12).

\begin{tabular}{|c|c|c|c|c|}
\hline \multirow{2}{*}{ Item } & \multirow{2}{*}{ Score } & \multicolumn{3}{|c|}{ Group } \\
\hline & & $\mathrm{S}$ & M & $\mathrm{L}$ \\
\hline \multirow[t]{4}{*}{ Steatosis } & 0 & 0 & 0 & 0 \\
\hline & 1 & 0 & 0 & 0 \\
\hline & 2 & 0 & 0 & 1 \\
\hline & 3 & 6 & 6 & 5 \\
\hline \multirow[t]{4}{*}{ Lobular inflammation } & 0 & 0 & 0 & 0 \\
\hline & 1 & 2 & 1 & 1 \\
\hline & 2 & 1 & 4 & 2 \\
\hline & 3 & 3 & 1 & 3 \\
\hline \multirow[t]{3}{*}{ Hepatocyte ballooning } & 0 & 6 & 6 & 5 \\
\hline & 1 & 0 & 0 & 1 \\
\hline & 2 & 0 & 0 & 0 \\
\hline \multirow{3}{*}{$\begin{array}{l}\text { NAFLD activity score } \\
\text { (NAS)* }\end{array}$} & $0-2$ & 0 & 0 & 0 \\
\hline & $3-4$ & 2 & 1 & 1 \\
\hline & $5-8$ & 4 & 5 & 5 \\
\hline \multirow[t]{5}{*}{ Fibrosis } & 0 & 0 & 0 & 0 \\
\hline & 0.5 & 2 & 4 & 0 \\
\hline & 1 & 2 & 2 & 1 \\
\hline & 1.5 & 0 & 0 & 4 \\
\hline & 2 & 2 & 0 & 1 \\
\hline
\end{tabular}

Values indicate the number of rats.

* NAS scores of 5-8 were considered to be diagnostic for NASH, and scores of 0-2 were considered not to be diagnostic for NASH.

$\mathrm{S}$, rats fed a high-fat and high-cholesterol (HFC) diet from 5 to $14 \mathrm{wk}$ of age $(n=6)$; M, rats fed a HFC diet from 9 to 18 wk of age $(n=6)$; L, rats fed a HFC diet from 13 to 22 wk of age $(n=6)$.

and lowest in the L group from the start of HFC feeding up to $7 \mathrm{wk}$, and were still higher in the $S$ group than in the $\mathrm{M}$ and $\mathrm{L}$ groups at 8 and 9 wk after the start of HFC feeding (Fig. 1). After the 9-wk rearing period, the liver weight/body weight ratio was significantly higher in the $\mathrm{S}$ group than in the $\mathrm{L}$ group $(p=0.002)$, whereas the epididymal fat pad weight/body weight ratio did not differ significantly among the three groups (Table 3). Serum biochemical parameters and hepatic lipid concentrations

After the 9-wk rearing period, the serum levels of TG, FFA, TC, glucose, insulin and leptin, and serum activities of AST and ALT were not significantly different among the three groups. The serum adiponectin levels were significantly higher in the S group than in the $M$ and L groups ( $p=0.005$ and $p=0.001$, respectively). The hepatic TG and TC concentrations were not significantly different among the three groups, as determined by ANOVA and Tukey-Kramer's post hoc test (Table 3). Histopathological findings of the liver

Figure 2 shows representative histopathological find- ings in the liver of the S, M and L groups. The histopathological assessments after the 9-wk rearing period are shown in Table 4. Severe steatosis (score 3) was observed in all 6 rats in the S and M groups, respectively, and $5(83.3 \%)$ of the 6 rats in the L group. Moderate or severe lobular inflammation (score 2 or 3 ) was observed in $4(66.7 \%)$ of the 6 rats in the $S$ group and in 5 $(83.3 \%)$ of the 6 rats in the $\mathrm{M}$ and $\mathrm{L}$ groups, respectively. Hepatocyte ballooning was not observed in any of the rats except for 1 rat in the L group (score 1). According to the NAS score (12), $4(66.7 \%)$ of the 6 rats in the S group and $5(83.3 \%)$ of the 6 rats in each of the M and L groups were diagnosed with NASH. None of the above parameters were significantly different among the three groups, as determined by Fisher's exact probability test. Perisinusoidal and/or portal/periportal fibrosis (score 0.5 to 2) was observed in all rats, and the fibrosis scores 1.5 or 2 was significantly more seen in the L group ( 5 of 6 rats) than in the M group (0 of 6 rats), as determined by Fisher's exact probability test $(p=0.015)$. Rats' age (S, M and L groups) was not correlated with any of the histopathological assessments of the liver (score) such as hepatic steatosis, lobular inflammation, hepatocyte ballooning, NAS and fibrosis (score), as determined by Spearman's rank correlation coefficient $(r=-0.297$ and $p=0.231, r=0.077$ and $p=0.761, r=0.297$ and $p=0.231, \quad r=0.035$ and $p=0.891, \quad r=0.286$ and $p=0.250$, respectively).

Hepatic mRNA expression

As determined by ANOVA and Tukey-Kramer's post hoc test, the mRNA level of TGF- $\beta$ (Tgfb1), which is a key inducer of fibrogenesis, was significantly higher in the L group than in the $\mathrm{S}$ group $(p=0.021)$. The mRNA level of $\alpha$-SMA (Acta2), which is responsible for fibrogenesis, was highest in the L group and lowest in the $S$ group, although the difference was not statistically significant. The mRNA level of IL- $1 \beta(\operatorname{Illb})$, which is a pro-inflammatory cytokine, was also highest in the L group and lowest in the S group, although the difference was not statistically significant. The mRNA level of $\mathrm{NF}-\kappa \mathrm{B}(\mathrm{N} f \mathrm{~kb})$, which regulates inflammatory responses, was significantly higher in the M group than in the $\mathrm{S}$ group $(p=0.046)$. The mRNA level of HO-1 (Hmox 1$)$, which is an inducible enzyme that is activated in response to oxidative stress, was significantly higher in the $\mathrm{M}$ group and $\mathrm{L}$ group than in the $\mathrm{S}$ group ( $p=0.040$ and $p=0.012$, respectively). The mRNA level of MnSOD (Sod2), which is a member of the iron/manganese superoxide dismutase family, was significantly higher in the L group than the $\mathrm{S}$ group $(p=0.028)$. The mRNA level of CYP2E1 (Cyp2e1), which is a member of the cytochrome P450 mixed-function oxidase system, was highest in the L group, although the difference was not statistically significant. The mRNA level of GPX-1 $(G p x 1)$, which is an intracellular antioxidant enzyme, was highest in the L group and lowest in the $\mathrm{S}$ group, although the difference was not statistically significant. The mRNA levels of FAS (Fasn), GPAT1 (Gpam), CPT-1 (Cpt1a) and FXR (Nr1h4), which are involved in cholesterol or lipid metabolism, were highest in the L group 
A Fibrosis
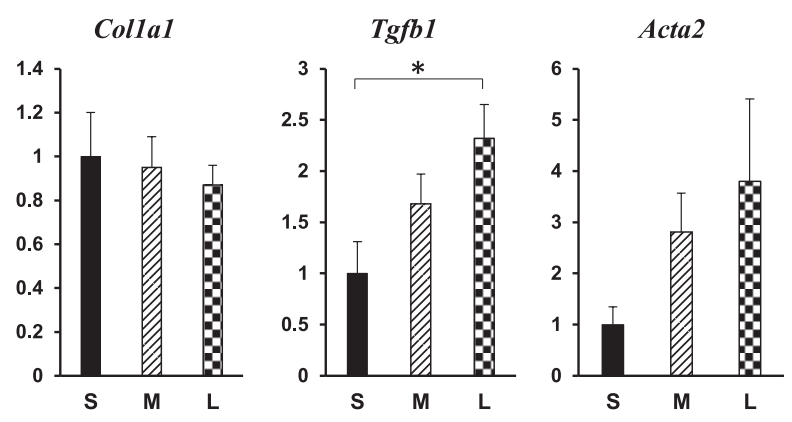

B Inflammation
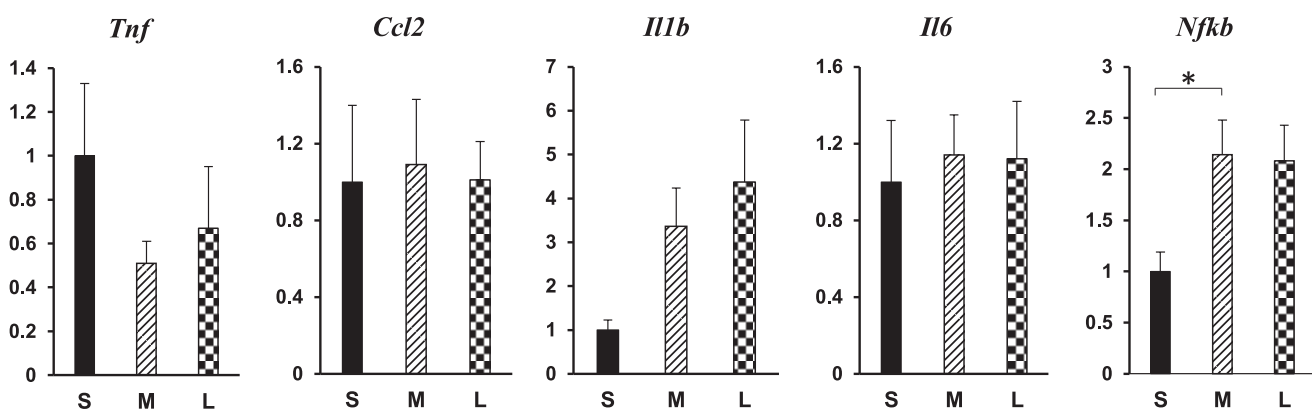

C Oxidative stress
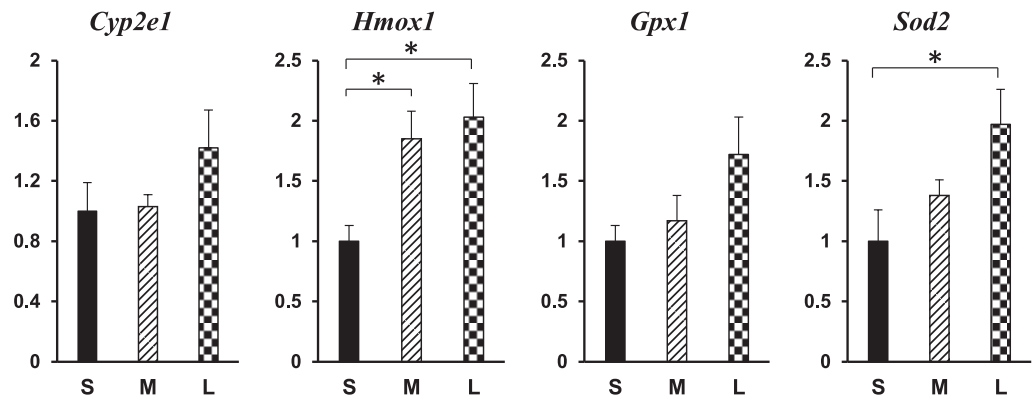

D Cholesterol or lipid metabolism
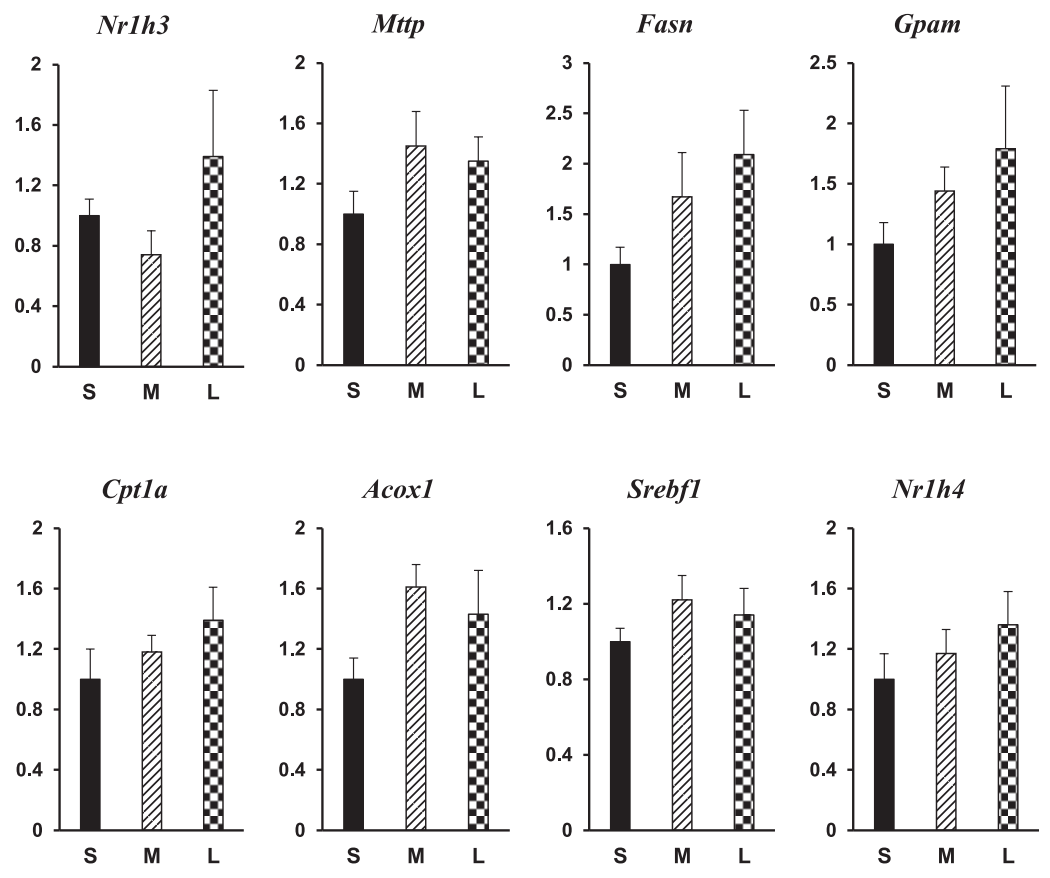
and lowest in the S group, although the difference was not statistically significant (Fig. 3).

\section{DISCUSSION}

In the present study, we evaluated the age-related alterations of NASH in 5-, 9- and 13-wk-old male SD rats that were fed a HFC diet for $9 \mathrm{wk}$. Male rats are weaned at an average age of $21 \mathrm{~d}$ ( $3 \mathrm{wk}$ ) after birth, and they begin to undergo sexual maturation at an average age of 45 to $48 \mathrm{~d}$ (6-7 wk) after birth. Adulthood, characterized by behaviors such as increased risk-taking and social play, begins approximately $8 \mathrm{wk}$ after birth (13). Therefore, the ages of 5, 9 and 13 wk of age in rats can be considered the equivalent of periadolescent, young adulthood and adulthood in humans, respectively.

The natural history of pediatric NAFLD remains unclear mainly due to the lack of longitudinal prospective studies focusing on its progression, and the progression of the disease is difficult to predict in children (1, 5, 7). Feldstein et al. reported that children with NAFLD had a 13.8-fold higher risk of dying or requiring liver transplantation than the age- and sex-matched general population (14). Thus, NAFLD/NASH in children may represent a more severe phenotype with increased morbidity and mortality in adulthood, indicating that early identification and management is needed $(5,14)$.

In the present study, the cumulative energy intake, body weight gain and food efficacy during the 9-wk rearing period were highest in the $S$ group and lowest in the L group; these may have been high in the $S$ group because the rats were at an age of rapid body growth. In an interventional study using adult rats, the cumulative energy intake and/or body weight gain should be equivalent among groups by dietary restriction in order to compare groups and interpret the nutritional effect. However, in SD rats, daily food intake has been observed to be largest between 7 to $10 \mathrm{wk}$ of age, and after then, this intake gradually decreased (personal communication from manufacturer). Because the present study was designed to examine the age-related (including eating habits) alterations of NASH in SD rats that were fed a same (HFC) diet for the same period ( $9 \mathrm{wk})$, all rats had free access to food and water. Therefore, daily food intake (g)/body weight (g) was highest in the S group (feeding between 5 to $14 \mathrm{wk}$ of age) and lowest in the L group (feeding between 13 to 22 wk of age) in most of the 9-wk rearing period, although the difference was getting closer over time in the present study. Indeed, daily food intake $(\mathrm{g}) /$ body weight $(\mathrm{g})$ was similar at $14 \mathrm{wk}$ of age rats in the $\mathrm{S}, \mathrm{M}$ and $\mathrm{L}$ group $(0.039 \pm 0.002$, $0.041 \pm 0.002,0.041 \pm 0.002$, respectively) as determined by ANOVA and Tukey-Kramer's post hoc test. Our results disclosed that NASH was not more evident in the $S$ group than the other two groups, despite the larger amounts of cumulative energy intake, body weight gain and daily food intake (g)/body weight $(\mathrm{g})$.

Serologically, almost all parameters were similar among the three groups, except for the serum adiponectin levels. Pubertal development can influence the serum adiponectin levels, and an age-dependent decrease of serum adiponectin levels has been reported in normalweight children $(15,16)$. Almost all histopathological findings were similar among the three groups in the present study, but the hepatic fibrosis score was significantly higher in the L group than in the M group, although rats' age (S, M and L groups) was not correlated with hepatic fibrosis (score), as determined by Spearman's rank correlation coefficient. Also, the mRNA levels of fibrosis-related genes encoding TGF- $\beta$ and $\alpha$-SMA were lowest in the $\mathrm{S}$ group and highest in the L group. Moreover, mRNA levels of inflammation-related genes encoding $\mathrm{IL}-1 \beta$ and $\mathrm{NF}-\kappa \mathrm{B}$, oxidative stress-related genes encoding CYP2E1, HO-1, GPX-1 and MnSOD, cholesterol or lipid metabolism-related genes encoding FAS, GPAT1, CPT-1 and FXR were highest in the L group and lowest in the $\mathrm{S}$ group, although the difference was not statistically significant. These results corroborated those of previous reports suggesting that the risk of NASH or NAFLD-related fibrosis increases with age $(2,3,7)$.

The present study had several limitations. First, we only evaluated rats that were relatively young because, although rats have an average life span of 2.5 to $3.5 \mathrm{y}$, skeletal growth tapers off at approximately 7 to 8 mo of age (13). Second, all rats were equally fed a HFC diet for 9 wk. However, in humans, the earlier unhealthy eating habits start, the longer such habits continue, which can lead to more serious outcomes in the development and progression of NAFLD/NASH. In this context, the cumulative energy intake and/or body weight gain should have been equivalent among groups by dietary restriction in order to interpret the exact nutritional effect in young rats.

In conclusion, our results indicated that age-related alterations of NASH were not serologically and histopathologically obvious in 5-, 9- and 13-wk-old male SD rats that were fed a HFC diet for $9 \mathrm{wk}$. However, the mRNA expression levels of some fibrogenic, inflammatory, oxidative stress and cholesterol or lipid metabolism-related genes in the liver suggested that aging is likely associated with the development of NASH. In contrast, NASH was not more evident in younger rat group, despite the larger amounts of cumulative energy intake, body weight gain and daily food intake (g)/body weight (g). Further studies with a longer HFC diet-feed-

Fig. 3. Hepatic expression of genes involved in (A) fibrosis, (B) inflammation, (C) oxidative stress, and (D) cholesterol or lipid metabolism in the $\mathrm{S}, \mathrm{M}$ and L groups ( $n=6$ /group) after the 9-wk rearing period. The mRNA levels are expressed relative to the levels of the $S$ group (means \pm SE). ${ }^{*} p<0.05$ as determined by one-way analysis of variance (ANOVA) and Tukey-Kramer's post hoc test. S, rats fed a high-fat and high-cholesterol (HFC) diet from 5 to 14 wk of age $(n=6)$; M, rats fed a HFC diet from 9 to $18 \mathrm{wk}$ of age $(n=6)$; L, rats fed a HFC diet from 13 to 22 wk of age $(n=6)$. 
ing period, especially in younger rats, are needed in the future.

\section{Disclosure of State of COI}

The authors declare no conflicts of interest.

\section{Acknowledgments}

This study was supported by research funds from the University of Nagasaki.

\section{REFERENCES}

1) Bush H, Golabi P, Younossi ZM. 2017. Pediatric nonalcoholic fatty liver disease. Children 4: 48.

2) Younossi ZM. 2018. Non-alcoholic fatty liver disease-a global public health perspective. J Hepatol. Advance Publication, doi: 10.1016/j.jhep.2018.10.033.

3) Sheedfar F, Di Biase S, Koonen D, Vinciguerra M. 2013. Liver diseases and aging: friends or foes? Aging Cell 12: 950-954.

4) Shima T, Seki K, Umemura A, Ogawa R, Horimoto R, Oya H, Sendo R, Mizuno M, Okanoue T. 2015. Influence of lifestyle-related diseases and age on the development and progression of non-alcoholic fatty liver disease. Нерatol Res 45: 548-559.

5) Smith SK, Perito ER. 2018. Nonalcoholic liver disease in children and adolescents. Clin Liver Dis 22: 723-733.

6) Anderson EL, Howe LD, Jones HE, Higgins JPT, Lawlor DA, Fraser A. 2015. The prevalence of non-alcoholic fatty liver disease in children and adolescents: a systematic review and meta-analysis. PLOS ONE 10: e0140908.

7) Doycheva I, Watt KD, Alkhouri N. 2017. Nonalcoholic fatty liver disease in adolescents and young adults: the next frontier in the epidemic. Hepatology 65: 2100-2109.

8) Nier A, Brandt A, Conzelmann IB, Ozel Y, Bergheim I. 2018. Non-alcoholic fatty liver disease in overweight children: role of fructose intake and dietary pattern. Nutrients 10: 1329.

9) Ichimura M, Kawase M, Masuzumi M, Sakaki M, Nagata Y, Tanaka K, Suruga K, Tamaru S, Kato S, Tsuneyama K, Omagari K. 2015. High-fat and high-cholesterol diet rapidly induces nonalcoholic steatohepatitis with advanced fibrosis in Sprague-Dawley rats. Hepatol Res 45: 458-469.

10) Ichimura M, Masuzumi M, Kawase M, Sakaki M, Tamaru S, Nagata Y, Tanaka K, Suruga K, Tsuneyama K, Matsuda S, Omagari K. 2017. A diet induced Sprague-Dawley rat model of nonalcoholic steatohepatitis-related cirrhosis. J Nutr Biochem 40: 62-69.

11) Folch J, Lees M, Sloane Stanley GH. 1957. A simple method for the isolation and purification of total lipids from animal tissues. J Biol Chem 226: 497-509.

12) Kleiner DE, Brunt EM, Van Natta M, Behling C, Contos MJ, Cummings OW, Ferrell LD, Liu Y-C, Torbenson MS, Unalp-Arida A, Yeh M, McCullough AJ, Sanyal AJ. 2005. Design and validation of a histological scoring system for nonalcoholic fatty liver disease. Hepatology 41: 1313-1321.

13) Sengupta P. 2013. The laboratory rat: relating its age with human's. Int J Prev Med 4: 624-630.

14) Feldstein AE, Charatcharoenwitthaya P, Treeprasertsuk S, Benson JT, Enders FB, Angulo P. 2009. The natural history of nonalcoholic fatty liver disease in children: a follow-up study for up to 20-years. Gut 58: 1538-1544.

15) Erhardt E, Foraita R, Pigeot I, Barba G, Veidebaum T, Tornaritis M, Michels N, Eiben G, Ahrens W, Moreno LA, Kovacs E, Molnar D, on behalf of the IDEFICS consortium. 2014. Reference values for leptin and adiponectin in children below the age of 10 based on the IDEFICS cohort. Int J Obes 38: S32-S38.

16) Lausten-Thomsen U, Christiansen M, Fonvig CE, Trier $C$, Pedersen O, Hansen T, Holm JC. 2015. Reference values for serum total adiponectin in healthy non-obese children and adolescents. Clin Chim Acta 450: 11-14. 